\title{
III. Die erste Stufe der Westhilfe: Der „Westgrenzfonds“
}

\section{Der Westgrenzfonds als einmalige Beihilfe}

Als am 9. Juli 1927 der Haushaltsausschuß des Reichstags über die endgültige Aufteilung der im Grenzprogramm 1927 bereitgestellten Gelder zu entscheiden hatte, stellte der Abgeordnete v. Guérard im Namen der Regierungsparteien den Antrag, den Vorschlag des Innenministers nur unter der ausdrücklichen Feststellung zu billigen, daß der Westen ,trotz gleichartiger Verhältnisse" nicht berücksichtigt worden sei. Die Zustimmung sei obendrein mit der Forderung zu verknüpfen, daß entweder im Nachtrag für den Etat 1927 oder im Haushalt 1928 entsprechende Mittel für die Westgebiete ausgewiesen würden. ${ }^{1}$ Da noch vor der Aussprache Finanzminister Köhler seine Bereitschaft erkennen ließ, ,diesem Wunsche zu entsprechen“, zeigte sich die Opposition dem Antrag gegenüber gespalten. Der Sprecher der SPD widersetzte sich dem Begehren v. Guérards, da er eine weitere Verwässerung der Grenzhilfe befürchtete, die ja ursprünglich nur auf den besonders bedürftigen preußischen Osten ausgerichtet gewesen sei. Der Vertreter der DDP erklärte sich mit dem Antrag jedoch unter der Bedingung einverstanden, daß die finanzielle Förderung des Westens die Mittel für die Osthilfe nicht schmälern dürfe, was ihm v. Guérard als selbstverständlich zugestand. Daraufhin verabschiedete die Mehrheit im Haushaltsausschuß eine Entschließung über die Einrichtung einer Westhilfe. ${ }^{2}$ Die Höhe des zukünftigen Westfonds handelte v. Guérard persönlich mit dem Finanzminister aus, der schließlich zusicherte, er werde die dem Rheinministerium bereits bewilligten 3 Millionen RM um weitere 9,5 Millionen aufstocken und den Gesamtbetrag von 12,5 Millionen im Nachtragsetat 1927 unter dem Einzelhaushalt des Innenministeriums ausbringen. ${ }^{3}$ Außerdem rang er dem Minister noch das Zugeständnis ab, daß diese Mittel bereits vor der endgültigen Verabschiedung des Nachtragshaushaltes ausgegeben werden durften. ${ }^{4}$

Sowohl die konjunkturelle Entwicklung der deutschen Volkswirtschaft wie auch die politische Lage begünstigten v. Guérards Bestrebungen. Im Juli 1927 durchlief die Wirtschaft gerade eine Phase des Aufschwungs, und die Zahl der Arbeitslosen sank zum ersten Mal seit Oktober 1925 wieder unter die Grenze von 5\% der erwerbsfähigen Bevölkerung. ${ }^{5}$ Während mithin die

${ }^{1}$ BA R 43 I/1798, Bl. 155.

2 Ebd.

3 Ebd., Bl. 147.

4 BA R 43 I/1799, BI. 105.

5 Vgl. D. Keese, Die volkswirtschaftlichen Gesamtgrößen für das Deutsche Reich in den 
konjunkturbedingten sozialen Ausgaben des Staates schrumpften, stiegen dessen Steuereinkünfte an. Da überdies im Frühjahr 1928 Neuwahlen zum Reichstag bevorstanden, die es vielen Abgeordneten ratsam erscheinen ließen, sich durch Wahlgeschenke beim Wähler zu empfehlen, erreichte die Bewilligungsfreudigkeit auch in den Reihen jener Parteien einen Höhepunkt, die sonst einer Ausweitung der Staatstätigkeit - wie etwa die DDP oder einer Ausuferung des Subventionswesens - wie etwa die SPD - skeptisch gegenüberstanden. ${ }^{6}$ Daher erstaunt es nicht, daß der Haushaltsausschuß den Vorschlag des Finanzministers annahm. Allerdings sollten aus dem Fonds von 12,5 Millionen RM, der schon bald die amtliche Bezeichnung „Westgrenzfonds“ (WGF) erhielt, Unterstuitzungen in Höhe von 2 bis 2,5 Millionen zugunsten der ,Saargänger" abgezweigt werden. ${ }^{7}$ Bei den Saargängern, die für die künftige Gestaltung der Westhilfe noch eine Rolle spielen sollten, handelte es sich um Bewohner der dem Saargebiet benachbarten deutschen Gemeinden, die entweder das ganze Jahr hindurch oder in der Winterszeit täglich oder wöchentlich von ihren Heimatorten aus zur Arbeit an die Saar fuhren. An ihren Arbeitsplätzen im Saargebiet wurden sie mit französischen Franken entlohnt, deren Kaufwert im Vergleich zur Reichsmark mit dem Beginn der Inflation in Frankreich etwa ab 1925 ständig schwand. Um ihnen dennoch den Ertrag ihrer Arbeit zu sichern, gewährte ihnen die Reichsregierung Ausgleichszahlungen. ${ }^{8}$ Zu Beginn des Jahres 1927 hatte Staatssekretär Schmid angeregt, diese Hilfsaktion, die besonders den Einzelhaushalt des Rheinministeriums belastete, allmählich abzubauen, da die Währung Frankreichs inzwischen saniert und gefestigt sei. ${ }^{9}$ Gegen dieses Vorhaben hatte aber sogleich Reichsarbeitsminister Brauns Widerspruch eingelegt, wobei er sich auf das Fehlen geeigneter Arbeitsplätze im Grenzgebiet und die hohen Aufwendungen dieser Arbeiter für die Anfahrt und die Unterkunft berufen hatte. Deshalb hatte erst am 1. Juli der Ausschuß für die besetzten Gebiete ausdrücklich auf der Fortsetzung der ,Saargängerunterstützung " bestanden. ${ }^{10}$

Als im August die Beamten im Reichsinnenministerium mit der Aufstellung eines Verteilungsplanes für den ,"neuen “ Westgrenzfonds begannen ${ }^{11}$, hoffte Rheinminister Marx, die Länder würden sich unmittelbar an der Durchfüh-

Jahren 1925-1936, in: W. Conze u. a. (Hg.), Die Staats- und Wirtschaftskrise des Deutschen Reichs 1929/33, 1967, S. 35 f.

6 Vgl. D. Baumgarten, Deutsche Finanzpolitik 1924-1928, 1965, S. 129, 193.

7 BHStA MA 107776, 12.7.27.

8 Vgl. S. Wambaugh, The Saar Plebiscite. With a Collection of Official Documents, $1971^{2}$, S. 13.

9 GLA 233, Nr. 12073, 4.2.27; BA R 43 I/1420, Bl. 129.

10 Ebd., 1.7.27; ebd., Bl. 129.

11 BA R 43 I/1798, Bl. 149. 
rung eines Westprogramms beteiligen. Die Verhandlungen, die sein Staatssekretär Schmid anläßlich einer Informationsreise in die Bezirke der Westgrenze führte, erbrachten jedoch kein greifbares Ergebnis. Insbesondere Preußens Finanzminister Höpker-Aschoff schärfte allen preußischen Ressorts seinen Standpunkt ein, ,daß die Beseitigung der durch die Kriegs- und Friedensvertragsfolgen geschaffenen Notstände und Schäden ausschließlich Sache des Reiches" sei. Úberdies richtete er an Schmid die Gegenfrage, ob der Staatssekretär bei seinen Besprechungen mit Regierungsvertretern ,in gleicher Weise" wie in den preußischen Grenzzonen auch in der Pfalz die Ausschüttung der Reichshilfe von einer zusätzlichen Bereitstellung bayerischer Gelder abhängig gemacht habe. ${ }^{12}$ Daher konnte man sich im Rheinministerium ausrechnen, daß die bayerische Staatsregierung einen entsprechenden Schritt Preußens als Bedingung für ihre Mitarbeit voraussetzen würde. Mithin war der ursprüngliche Plan des Ministers für die besetzten Gebiete, mit den Subventionen aus der Reichskasse den Grundstein für ein von den beteiligten Ländern getragenes Westprogramm zu legen, gescheitert.

Deshalb teilte Marx am 3. November den Landesregierungen in München, Berlin und Oldenburg mit, das anläßlich der „Grenzbereisung“ Schmids erörterte Programm solle nunmehr als ,einmalige außerordentliche Beihilfe“ für wirtschaftliche oder kulturelle Maßnahmen „,beschleunigt zur Durchführung" gelangen. Eine gewisse Enttäuschung über die mangelnde Bereitschaft der Länder zur Mitarbeit an einem Westprogramm schlug sich in drei Bestimmungen nieder, die an die Vergabe der Gelder geknüpft wurde, nämlich:

1. Die Beihilfe des Reiches sei nicht dazu bestimmt, den ordentlichen Haushalt der beteiligten Länder zu entlasten.

2. Die Verwendung der Beihilfe müsse auch dann, wenn es sich um verhältnismäßig kleine Geldbeträge handle, ,,beschränkt bleiben auf die Schaffung dauernder, für die Hilfe des Reiches sichtbares Zeugnis ablegender Werte".

3. Die Einrichtung oder der Ausbau staatlicher Verwaltungsgebäude dürfe aus den Mitteln des Westgrenzfonds nicht bestritten werden. ${ }^{13}$

In der Anlage forderte Marx sodann die Regierungspräsidenten auf, ihm bis zum 26. November nach einem bestimmten Muster Anträge zur Bewilligung von Vorhaben einzureichen. Als obere Grenze für die Beantragung von Mitteln setzte er für Preußen 12, für die bayerische Rheinpfalz 6 und für das oldenburgische Birkenfeld 0,5 Millionen RM fest. Die Verteilung des Fonds werde dann in Verhandlungen mit den Ländervertretern nach dem Maßstab der ,,Reichswichtigkeit der angemeldeten Vorhaben“ erfolgen. ${ }^{14}$

12 GStA Rep. 90, Nr. 1125, 10.10.27.

13 BA R 43 I/1799, Bl. 108; BHStA MK 15538, 3.11.27.

14 Ebd. 
Dieses Verfahren sicherte dem Gedanken der Westhilfe eine günstige Ausgangsposition für die unmittelbar bevorstehenden Beratungen des Reichstags über die endgültige Verabschiedung des Nachtragsetats 1927. Die Regierungspräsidenten waren nämlich gezwungen, kurzfristig begründete Anträge einzureichen, deren Kostenvoranschläge die 12,5 Millionen des Westgrenzfonds, die überdies noch die Saargängerunterstützung einschlossen, bei weitem überstiegen. Ausgestattet mit Forderungen nach einer erheblich höheren Summe - allein die bayerische Rheinpfalz hatte Ansprüche über 7,5 Millionen gestellt ${ }^{15}$ - war Reichskanzler und Rheinminister Marx in der Lage, die Interessen des Westens zu behaupten, falls es zu einer erneuten Aufrechnung über die Hilfsbedürftigkeit der Ost-, Nord- und Westgrenze kommen sollte. ${ }^{16}$ Aus zwei Gründen war sogar zu erwarten, daß die Abgeordneten wieder versuchen würden, sich einander in der Schilderung der Notlage des jeweils von ihnen vertretenen Grenzdistrikts zu überbieten. Zum einen hatte sich die Reichsregierung nach der umstrittenen Aufteilung des Grenzprogramms 1927 verpflichtet, bei der Durchführung künftiger Grenzhilfen den Osten wieder stärker zu berücksichtigen. ${ }^{17}$ Zum anderen schürte der schlechte Ausfall der Ernte im Herbst 1927 die Unruhe unter den Landwirten, die vor allem in Schleswig-Holstein, Pommern und Ostpreußen die Forderung nach sofortigen Hilfsmaßnahmen des Reiches erhoben. ${ }^{18}$ Das Rheinministerium erhielt jedoch Schützenhilfe aus den Reihen derjenigen Parteien, die sich von einem Eintreten für die Lösung des Westproblems bei den kommenden Reichstagswahlen eine Verbesserung ihres Wahlergebnisses im Westen erhofften. Die Fraktionen des Zentrums, der BVP, der DNVP, der DDP und der SPD richteten nämlich Interpellationen an die Reichsregierung, die sich mit den wirtschaftlichen Schwierigkeiten einzelner Landschaften und einzelner Wirtschaftszweige des besetzten Gebietes beschäftigten. ${ }^{19}$ Obwohl sich schon am zweiten Verhandlungstag die Diskussion im Plenum fast ausschließlich den Schwierigkeiten der Ostprovinzen zuwandte, räumten kundige Beobachter ein, diese Serie parlamentarischer Anfragen habe immerhin bewirkt, daß die Anliegen der Westgrenze zum ersten Mal im Reichstag gebührend vertreten worden seien. ${ }^{20}$

Da außerdem der Haushaltsausschuß einen Betrag von 0,5 Millionen RM als ausreichend für die Unterstützung der Saargänger ansah ${ }^{21}$, verfügte Marx

15 BHStA MA 107776, 13. 1.28; MK 15538, 16.1.28.

16 Vgl. ebd. MK 15538, 5.11., 11.11.27.

17 Vgl. Hertz-Eichenrode, S. $218 \mathrm{f}$.

18 G. Stoltenberg, Politische Strömungen im schleswig-holsteinischen Landvolk 1918-1933. Ein Beitrag zur politischen Meinungsbildung in der Weimarer Republik, 1962, S. 107.

19 RT 419, Nr. 3694; RT 420, Nr. 3725, 3726, 3728, 3738, ferner PLT Drs. 14, Nr. 7279.

20 BHStA MA 107776, 7. 12.27.

21 Ebd. MK 15 538, 7.2. 28. 
über einen Fonds von 12 Millionen zur Durchführung wirtschaftlicher und kultureller Hilfsmaßnahmen im Westen. Somit gab das Rheinministerium am 13. Februar 1928 die endgültige Verteilung des Westgrenzfonds auf die beteiligten Länder bekannt: Preußen sollte 8, Bayern 3,5 und Oldenburg 0,2 Millionen RM erhalten. Eine Reserve von 300000 RM verblieb beim Ministerium. ${ }^{22}$ Diese Aufteilung löste den Widerspruch der bayerischen Staatsregierung aus, deren Vertreter in Berlin, Ministerialrat Sperr, sich schon seit geraumer Zeit um den Nachweis bemühte, daß das Ministerium für die besetzten Gebiete die preußische Grenzzone der Rheinpfalz gegenüber bevorzuge. ${ }^{23}$ Sperrs unablässigen Bemühungen, einen höheren Anteil für die Pfalz herauszuholen, vermochte Schmid auf die Dauer nicht zu widerstehen, obwohl er zunächst erklärt hatte, schon mit den 3 Millionen RM, die sein Referent ursprünglich vorgeschlagen habe, seien die bayerischen Projekte schon reichhaltig bedacht worden. ${ }^{24}$ Also gab er Sperrs Drängen nach und schlug die Reserve dem Anteil Bayerns zu, der sich damit auf 3,8 Millionen erhöhte. Von der Quote Preußens könne er nichts mehr abziehen, erklärte er Sperr, ,schon um in dieser politisch unruhigen Zeit - einem Wunsche des Reichskabinetts entsprechend - Preußen nicht zu vergrämen. “25 Tatsächlich benötigte die preußische Staatsregierung ihren Anteil dringend, um die Erbitterung unter den Kleinbauern, Winzern und dem „kleineren Mittelstand“ in der südlichen Rheinprovinz zu dämpfen, die sich dort angesichts der großzügigen und schnellen Hilfsmaßnahmen zugunsten Ostpreußens entzündet hatte. ${ }^{26}$

Andererseits kämpften Preußen und Bayern inzwischen gemeinsam im Reichsrat um eine Fortführung der Grenzhilfe. In diesem Gremium verfügten die preußische und die bayerische Regierung zusammen über eine Mehrheit von 37 von insgesamt 66 Stimmen. ${ }^{27}$ Im Dezember 1927 beriet der Reichsrat über den Haushaltsvoranschlag für das Rechnungsjahr 1928. Die Gesetzesvorlage sah keine besonderen Grenzprogramme mehr vor, nachdem sich sowohl Reichsfinanzminister Köhler wie auch Reichsinnenminister v. Keudell gegen eine Verlängerung der Grenzhilfen ausgesprochen hatten. ${ }^{28}$ Preußen beantragte indessen, auch in den Haushalt 192820 Millionen RM für die Grenzlandpolitik im Osten einzustellen, während Bayern 6 Millionen für die gesamte Westgrenze verlangte und außerdem eine weitere Unterstützung der Saargänger mit 1,5 Millionen und die Beibehaltung des 1927 erst-

22 Ebd. 13.2. 28.

23 Ebd. 8.11.27, 13.2., 16.2.28; MA 107776, 10.12.27, 27.2.28.

24 Ebd. 16.2., 27.2. 28.

25 Ebd., 17.2.28.

26 GStA Rep. 90, Nr. 1126, 29.2.28.

27 G.-J. Rose, Der Reichsrat der Weimarer Republik, 1964, S. 95.

28 BA R 43 I/1428, Bl. 232; BHStA MK 15538, 30.3. 28. 
malig eingerichteten Fonds von einer Million .,zur Förderung von Wirtschaft und Arbeit im besetzten Gebiet ${ }^{\star 29}$ empfahl. Alle Anträge fanden Mehrheiten. ${ }^{30}$

Diese Beschlüsse des Reichsrates legte die Reichsregierung gemeinsam mit ihrer Vorlage dem Reichstag zur endgültigen Entscheidung vor, der sich kurz vor dem Ende seiner Legislaturperiode im Frühjahr 1928 zu einem Kompromiß aufraffte. Auf der einen Seite lehnte er nämlich eine Weiterführung der Grenzprogramme im Haushalt 1928 ab, auf der anderen gestand er jedoch eine Erhöhung der im Nachtragshaushalt 1927 ausgebrachten finanziellen Mittel zu, wobei er auch den Westgrenzfonds von 12,5 auf 15 Millionen aufstockte. ${ }^{31}$ Diese Zulage von 2,5 Millionen, die jetzt neu verteilt werden mußte, bezeichnete die Ministerialbürokratie künftig als „Westgrenzfonds II"“. ${ }^{32}$ Schon im Reichsrat und erst recht im Reichstag waren Stimmen laut geworden, die eine Beteiligung Badens und Hessens an der Aufteilung dieses Fonds verlangten. ${ }^{33}$ Diese Forderung verwandelte den Westgrenzfonds II in einen politischen Zankapfel zwischen Reich und Ländern und verlieh ihm auf der Ebene der Innenpolitik einen Stellenwert, der seiner dürftigen materiellen Ausstattung nicht entsprach.

\section{Die Auseinandersetzung um die Beteiligung Badens und} Hessens an der Westhilfe

Überdies änderte die Reichstagswahl vom 20.5.1928 das politische Kräfteverhältnis. Aus diesen Wahlen gingen die Sozialdemokraten als Sieger hervor. Mit 153 Mandaten fiel ihnen als der nunmehr stärksten Fraktion fast ein Drittel der Sitze im Reichstag zu, während die bürgerlichen Parteien, und hier insbesondere das Zentrum und die DNVP, empfindliche Verluste hinnehmen mußten. ${ }^{34}$ An die Stelle des Bürgerblocks trat nun ein Kabinett der großen Koalition, gebildet aus SPD, Zentrum, BVP, DDP und DVP und geführt von dem Sozialdemokraten Hermann Müller. Auch für die Grenzlandpolitik erbrachte die Neubildung der Regierung bedeutsame personelle Veränderungen. Drei Reichsministerien, die bisher bei der Vorbereitung und

29 Dieser 1927 in den Haushalt des Rheinmınisteriums eingestellte Fonds diente vor allem der Förderung des fachlichen und beruflichen Verbandswesens in der Landwirtschaft und im Gewerbe ,,als Ansporn für den Fortschritt und zur Stärkung des Selbsthilfegedankens“. GStA Rep. 90, Nr. 1125, 28.5.27.

30 BHStA MA 107776, 16. 12.27; MA 107778, 12.9.28.

31 BA R 43 I/1799, Bl. 105, 110; GStA Rep. 90, Nr. 1126, 29.6. 28.

32 GStA Rep. 90, Nr. 1126, 3. 8.28; BHStA MK 15 539, 3.8. 28.

33 Vgl. BA R 43 I/1799, 19.7.28.

34 A. Milatz, Wähler und Wahlen in der Weimarer Republik, 1968, S. 89, 95, 105. 
bei der Durchführung der Grenzhilfen zusammengewirkt hatten, wurden jetzt von der SPD besetzt: Carl Severing, der bisherige preußische Innenminister, übernahm das Innenministerium, Rudolf Wissell löste Brauns als Arbeitsminister ab und Rudolf Hilferding wurde Finanzminister. Diese Neubesetzung erleichterte die Zusammenarbeit zwischen dem Reich und Preußen, denn wichtige Anliegen der preußischen Regierung konnten nun mit den sozialdemokratischen Reichsministern vorab geklärt werden. Dadurch gewann die preußische Staatsregierung in ihren Beziehungen zur Reichsregierung gegenüber allen anderen Ländern zu ihrem geographischen Vorteil auch noch einen zeitlichen Vorsprung. ${ }^{35}$ Die Zentrumsfraktion beschloß, lediglich v. Guérard „,als Verbindungsmann in ein Übergangskabinett“ zu entsenden ${ }^{36}$, wo er zunächst das Verkehrsministerium und das Ministerium für die besetzten Gebiete zugewiesen bekam.

In seiner Regierungserklärung entbot Reichskanzler Müller am 3. Juli „der treuen Bevölkerung an Rhein und Saar“" ,unseren herzlichen Gruß“. Er versicherte, die Reichsregierung sei sich der schweren Not bewußt, die in diesen Gebieten infolge der Besetzung herrsche und die durch die neue Grenzziehung noch verschärft werde, und er versprach, sein Kabinett werde vor allem den wirtschaftlichen Schwierigkeiten besondere Aufmerksamkeit widmen. ${ }^{37}$ Im Schatten dieser unverbindlichen, aber im Westen hochgespannte Erwartungen auslösenden Ankündigungen fiel dem Reichsminister für die besetzten Gebiete die schwierige Aufgabe zu, die 2,5 Millionen RM des Westgrenzfonds II an die Länder zu verteilen. Außerdem mußte v. Guérard den Vertretern der Landesregierungen mitteilen, der Reichstag empfehle, auf jeden Fall Hessen, vielleicht aber auch Baden an diesem Fonds zu beteiligen. Mit Rücksicht auf die künftig an den Reichstag zu stellenden Forderungen nach Grenzhilfe sei es taktisch klug, gab er dabei zu bedenken, Hessen und Baden jetzt nicht auszuschließen. Sodann begann er, die finanziellen Probleme des Volksstaates Hessen darzulegen, die er mit den Rückwirkungen des neuen Grenzverlaufs im Westen auf die hessische Wirtschaft begründete. Schließlich ließ er durchblicken, daß er der Regierung in Darmstadt bereits Zusagen über eine Beteiligung an der Westhilfe gemacht habe. ${ }^{38}$

Die Anstrengungen v. Guérards, Hessen unter allen Umständen in den Rang eines Grenzlandes zu erheben, wurzelten in dem besonderen Interesse seines Ministeriums und darüber hinaus der gesamten Reichsregierung, mit diesem Lande gute Beziehungen zu unterhalten. Schon unter Reichskanzler Marx

35 Rose, S. 96.

${ }^{36}$ O. Wachtling, Joseph Joos. Journalist, Arbeiterführer, Zentrumspolitiker. Politische Biographie 1878-1933, 1974, S. 124/125.

37 RT 423, S. 38/39.

${ }^{38}$ BHStA MA 107778, 12.9.28; MK 15539, 17.7.28. 
hatte das Rheinministerium mit der hessischen Landesregierung gut zusammengearbeitet. ${ }^{39}$ Darüber hinaus fiel Hessen innerhalb der Reichsländer die Rolle eines Vermittlers zwischen Nord und Süd zu. Sein langjähriger Staatspräsident Dr. Adelung (SPD) verstand es nämlich, sowohl mit Bayern als auch mit Preußen in freundschaftlichem Einvernehmen zu leben und auf den Länderkonferenzen die Gegensätze zwischen Unitarismus und Föderalismus zu mildern..$^{40}$ Außerdem waren die hessischen Staatsfinanzen derart zerrüttet, daß sie schon seit 1925 durch Zuweisungen der Reichsregierung saniert werden mußten. Für die Rechnungsjahre 1926 und 1927 hatte das Reich der Regierung in Darmstadt erhebliche Kassenvorschüsse bewilligt, die es in Zuschüsse umzuwandeln versprach, falls es die Úberzeugung gewinnen sollte, daß die besondere Notlage des Landes, ,im wesentlichen auf die Folge der Besatzung und des Ruhrkampfes" zurückzuführen sei. ${ }^{41}$ Mit dem Hinweis auf die Schäden, welche die Besatzung der wirtschaftlichen Entwicklung Hessens zugefügt habe und immer noch zufüge, hatte erst unlängst Staatspräsident Adelung den Anspruch seines Landes auf Grenzhilfe im hessischen Landtag bekräftigt ${ }^{42}$, während gleichzeitig der hessische Vertreter im Reichsrat die Beteiligung seines Landes am Westgrenzfonds I verlangt hatte. ${ }^{43}$ Aus allen diesen Gründen hielt v. Guérard den Versuch für geboten, Hessen wenigstens einen Anteil am Westgrenzfonds II zu verschaffen. Die Vertreter Preußens und Bayerns verschlossen sich jedoch solchen Úberlegungen. Sie beharrten auf dem Standpunkt, daß es sich bei den zur Debatte stehenden 2,5 Millionen RM um einen Fonds zur Förderung von Grenzgebieten handle, die Hessen nun einmal nicht vorzuweisen habe. ${ }^{44}$

Dieser Widerstand veranlaßte v. Guérard, eine Entscheidung des Reichskabinetts herbeizuführen. In der Sitzung vom 23.7. stellte er den Antrag, sowohl Hessen wie auch Baden eine Quote aus dem Westgrenzfonds II zu gewähren..$^{45}$ Unterstützung fand er jedoch nur bei Müller und Severing, die beide seine Auffassung teilten, die hessische Landesregierung dürfe in ihrer jetzigen Situation nicht den Eindruck gewinnen, als wolle sie das Reich im

39 Vgl. L. Bergsträsser (Hg.), Carl Ulrich. Erinnerungen des ersten hessischen Staatspräsidenten, 1953, S. 182.

40 B. Habel, Verfassungsrecht und Verfassungswirklichkeit. Eine Untersuchung zum Problem Reich - Länder, gezeigt am Beispiel Bayerns unter dem Kabinette Held (1924-1933), 1968, S. 35.

41 Vgl. Hess. StA Abt. 024 Hesse, Nr. 31, Ergebnisniederschrift S. 2.

42 B. Adelung, Sein und Werden. Vom Buchdrucker in Bremen zum Staatspräsidenten in Hessen, 1952, S. 303, 305.

43 RR 1928, S. 40.

${ }^{44}$ BHStA MK 15539, 17.7.28.

45 BA R 43 I/1799, Bl. 105f., 116/117. Zur folgenden Diskussion siehe auch ARWR, Das Kabinett Müller II, Bd. 1, 1970, S. 52-54. 
Stich lassen. Reichssparkommissar Saemisch ${ }^{46}$ erhob allerdings Einspruch. Nach seinen Erfahrungen sei das Defizit im hessischen Staatshaushalt nicht durch Grenzschäden verursacht worden; wolle man nun aber wegen dieses Fehlbetrages Hessen Gelder zuwenden, deren Verwendung im Grenzgebiet das Haushaltsrecht zwingend vorschreibe, so würden andere Länder ähnliche Forderungen von unübersehbarer finanzieller Tragweite stellen. Hilferding, der am 11. Juli eine eingehende Unterredung mit Adelung über die finanziellen Schwierigkeiten Hessens gehabt hatte ${ }^{47}$, stimmte Saemisch zu und empfahl die Ablehnung des Antrags. Wissell schloß sich mit dem aus der Sicht des Reichsarbeitsministers verständlichen Argument an, bei der Geringfügigkeit der noch verfügbaren Mittel könne den hessischen Staatsfinanzen ohnehin nicht wirksam geholfen werden, also sei es besser, die 2,5 Millionen des Westgrenzfonds II zur Weiterführung der in der Grenzzone bereits begonnenen Bauvorhaben zu verwenden. Das Votum des Reichsfinanzministers dürfte den Ausschlag dafür gegeben haben, daß das Reichskabinett in einer Abstimmung die Beteiligung Hessens am Westgrenzfonds II verwarf, ohne zuvor noch die Frage eines badischen Anteils zu erörtern. ${ }^{48}$ Daraufhin verteilte v. Guérard den Betrag von 2,5 Millionen RM nach dem Verteilungsschlüssel des Westgrenzfonds I. Nach Abzug einer Reserve von 300000 RM entfielen auf Preußen 1,46 Millionen, auf Bayern 700000 RM und auf Oldenburg 40000 RM. ${ }^{49}$

Die hessische Landesregierung nahm diese Entscheidung widerspruchslos hin, zumal Hilferding Staatspräsident Adelung weitere Zwischenkredite des Reiches in Aussicht gestellt hatte. ${ }^{50}$ Die Regierung Badens protestierte zwar gegen diese Aufteilung, wobei sie erneut die Bedeutung der früher bestehenden engen Verbindung ihrer Wirtschaft mit der des jetzt zu Frankreich gehörenden Elsaß unterstrich, doch maß v. Guérard diesem Einspruch zunächst keine große Bedeutung bei. ${ }^{51}$ Im Herbst freilich nahmen zwei badische Zentrumspolitiker den Kampf mit der Reichsregierung um eine Einstufung Badens als Grenzland auf. In einer Pressekampagne beschuldigten Dr. Joseph Schofer, der Führer des badischen Zentrums ${ }^{52}$, und der einen badischen Wahlkreis vertretende Reichstagsabgeordnete Joseph Ersing, ein christlicher

$46 \mathrm{Zu}$ den Aufgaben des Reichssparkommissars siehe Horkenbach, C. (Hg.), Das Deutsche Reich von 1918 bis heute, Bd. 1, 1930, S. 568.

47 Vgl. Hess. StA Abt. 024 Hesse, Nr. 31.

48 BA R 43 I/1799, Bl. 118, ferner BHStA MA 107 778, 12.9. 28.

${ }^{49}$ BHStA MK 15539, 3. 8. 28; GStA Rep. 90, Nr. 1126, 8. 8. 28.

50 Vgl. BA R 43 I/1799, Bl. 172; ARWR, Das Kabinett Müller II, Bd. 1, 1970, S. 27.

51 BA R 43 I/1799, Bl. 158, ferner ARWR, Das Kabinett Müller II, Bd. 1, 1970, S. 240, Anm. 19.

52 Vgl. P. Enderle, Dr. Joseph Schofer. „Der ungekrönte Großherzog von Baden“, Karlsruhe 1957. 
Gewerkschaftssekretär, das Reichskabinett, es benachteilige den Freistaat Baden bei der Verteilung der Grenzfonds. Gleichzeitig warfen sie der bayerischen Staatsregierung vor, daß sie trotz ihrer Lippenbekenntnisse zu den gemeinsamen Interessen Süddeutschlands gegen eine Bevormundung durch das ,große“ Preußen einträchtig mit preußischen Regierungsvertretern zusammenarbeite, um auf Kosten der süddeutschen Nachbarn Geschäfte zu machen. ${ }^{53}$ Mit diesen gezielten Angriffen erreichten die beiden Zentrumspolitiker zweierlei: Zunächst mußte sich Badens Staatspräsident Remmele dazu bequemen, sich in einem Schreiben an Reichskanzler Müller darüber zu beschweren, daß die Reichsregierung unter einem von der SPD gestellten Kanzler dem Land Baden mit einem gleichfalls sozialdemokratischen Staatspräsidenten Rechte versage, die es Preußen und Bayern ohne weiteres zugestehe. ${ }^{54}$ Außerdem aber fühlte sich Bayerns Ministerpräsident Heinrich Held zu der mehrmaligen ausdrücklichen Versicherung veranlaßt, nichts liege seiner Regierung ferner, als sich auf Kosten Badens zu bereichern, ganz im Gegenteil, die Vertreter Bayerns beim Reich seien angewiesen, sich stets für die badischen Interessen einzusetzen. ${ }^{55}$ Vor allem diese Zusicherung erleichterte es v. Guérard, einen für alle Seiten tragbaren Vergleich zustandezubringen. Unter Zustimmung der Landesregierung in Berlin und in München bot er Baden die Kassenreserve des Westgrenzfonds II, also 300000 RM, als Grenzhilfe an. ${ }^{56}$

\section{Der Westgrenzfonds als Instrument der Wirtschaftspolitik}

Der erbitterte Kampf um die Verteilung der 15 Millionen des Westgrenzfonds wirft die Frage nach der wirtschaftspolitischen Bedeutung dieser Regionalsubvention auf. Um deren Größenordnung etwas abschätzen zu können, sei zum Vergleich angeführt, daß im Reichshaushalt 1927 insgesamt 282,1 Millionen RM, im Etatjahr 1928 sogar 476,8 Millionen für die ,,inneren Kriegslasten" ausgewiesen waren. ${ }^{57}$ Zur Beantwortung dieser Frage soll zunächst einmal die Art der Verwendung dieser Mittel aufgezeigt werden, und zwar, bedingt durch die günstige Quellenlage, am Beispiel des bayerischen Anteils. ${ }^{58}$ Dieses Beispiel darf als repräsentativ betrachtet werden,

53 BA R 43 I/1799, Bl. 160, 163, 165; BHStA MA 107778, 3. 10., 4. 10. 28.

54 BA R 43 I/1799, Bl. 157.

55 BA R 43 I/1799, Bl. 162; BHStA MA 107778, 19.9., 12.10. 28.

56 Ebd., Bl. 172; ebd., 12.10., 17. 10. 28.

57 Siehe hierzu und auch zur Abgrenzung des Begriffs „Innere Kriegslasten“ F. Neumark, Kriegslasten und Reichshaushalt, in: Wirtschaftskurve 1930, S. 75.

58 Einer Schätzung des quantitativen Umfangs der Verwendung des Westfonds in den einzelnen Ländern steht bereits auf der Ebene des Reiches die Schwierigkeit entgegen, 
weil die von der Reichsregierung für die Grenzbezirke der Rheinpfalz bewilligten Projekte qualitativ weitgehend den Vorhaben entsprechen, die für Birkenfeld und für den Grenzgürtel der preußischen Rheinprovinz genehmigt worden waren. Auch hinsichtlich der quantitativen Aufteilung der Gelder gab es zwischen den Grenzlandschaften im Westen keine nennenswerten Unterschiede, denn zum einen wiesen diese Gebiete eine fast übereinstimmende wirtschaftliche und soziale Struktur auf, zum anderen aber hatte das Rheinministerium die hohen finanziellen Anforderungen, welche Bayern zunächst für religiöse und kulturelle Zwecke gestellt hatte, auf das Maß zurückgeführt, das diesem Bereich in den Anträgen Preußens zukam. ${ }^{59}$ Von den 4,5 Millionen RM, die Bayern aus dem Westgrenzfonds empfangen hatte, waren bis Ende 1932 verwendet worden: ${ }^{60}$

a) $33 \%$ für den Neu- und Ausbau von Kirchen und Pfarrhäusern, Schulhäusern und Lehrerwohnungen, Krankenhäusern, Kindergärten und Heimstätten für Schwerkriegsbeschädigte,

b) $25 \%$ für den Ausbau von Bezirksstraßen und Gemeindewegen,

c) $14 \%$ für die Landwirtschaft, und zwar insbesondere für die Errichtung von landwirtschaftlichen Schulen, die Förderung der Vieh- und Pferdezucht, der Grünlandwirtschaft, der Milchwirtschaft, des Obstbaues, der Flurbereinigung und der Bodenkultur,

d) $10 \%$ für den Ausbau der kommunalen Wasserversorgung,

e) $7 \%$ für Industrie, Handel und Gewerbe, insbesondere für die Förderung von Industrieunternehmungen.

Der Industrieförderung kam also innerhalb des Westgrenzfonds eine vergleichsweise geringe Bedeutung zu. Bewußt verzichteten das Reich und die Länder auf den Versuch, mit Hilfe staatlicher Subventionen an der Westgrenze Industriebetriebe anzusiedeln. Ausnahmen wurden nur dort zugelassen, wo sich wie z. B. in der Südpfalz einfach kein anderer Weg fand, um den „Elsaßgängern“, die ihre Arbeitsplätze im benachbarten Frankreich verloren

„daß es in der Statistik der Reichsschuld dieser Jahre von nachträglichen Berichtigungen, Umbuchungen und Änderungen der Statistik nur so wimmelt". R. E. Lüke, Von der Stabilisierung zur Krise, 1958, S. 92. Auch die Höhe der Zuwendungen für das Saargebiet läßt sich kaum endgültig ermitteln, ,zumal selbst die zuständigen Stellen wie das Saarreferat des Auswärtigen Amtes nur zu annähernden Schätzungen in der Lage waren". F. Jacoby, Die nationalsozialistische Herrschaftsübernahme an der Saar. Die innenpolitischen Probleme der Rückgliederung des Saargebietes bis 1935, 1973, S. 47. Für Bayern liegt jedoch die offenbar aus privater Initiative entstandene Zusammenstellung eines Verwaltungsbeamten vor: „Darstellung der gesamten Reichswesthilfe von 1928 bis einschließlich 1932, verfaßt von Reg.-direktor Staehler i. R., Speyer im Oktober 1933“. BHStA MWi 8246.

59 Vgl. BHStA MA 107776, 13.1.28; MK 15538, 1.2., 21.3.28.

${ }^{60}$ Berechnet nach den Zahlenangaben in Staehlers „Darstellung“. 
hatten, eine Erwerbsmöglichkeit zu schaffen. ${ }^{61}$ Abgesehen vom Widerstand, den alteingesessene Firmen Plänen der Ansiedlung von Konkurrenten entgegensetzen würden, schätzten Experten die Erfolgsaussichten von Industriesubventionen in den frachtungünstig gelegenen Grenzdistrikten als außerordentlich gering ein. ${ }^{62}$ Die Entscheidung des Rheinministeriums und der Landesregierungen, abweichend von dem bei der Osthilfe eingeschlagenen Weg die Mittel des Westgrenzfonds in erster Linie dazu zu benutzen, um das Bildungsangebot zu erweitern, die Infrastruktur zu verbessern, soziale Härten auszugleichen und über Bau- und Reparaturaufträge das ortsansässige Handwerk und Gewerbe zu beschäftigen, zeugt daher von volkswirtschaftlicher Einsicht.

Die Wirkungen, die von dieser Regionalsubvention auf den Arbeitsmarkt ausgingen, dürfen nicht an ihrer etatmäßigen Ausstattung mit 15 Millionen RM gemessen werden. Da es sich in der Regel bei den Einzelbewilligungen aus dem Westgrenzfonds um Zuschüsse für Projekte handelte, bei denen eine finanzielle Beteiligung der Gemeinden, der Verbände, der Landwirte oder der Unternehmer bindend vorgeschrieben war ${ }^{63}$, löste die Westhilfe eine weitaus größere Nachfrage aus. Den zahlreichen Eingaben um die Gewährung einer Beihilfe ist zu entnehmen, daß viele arbeitsintensive Bauvorhaben wohl nicht begonnen worden wären, wenn zu den vorhandenen finanziellen Mitteln nicht noch die Zuschüsse des Reiches geflossen wären. ${ }^{64} \mathrm{Au}$ Berdem bemühte sich der Reichstagsausschuß für die besetzten Gebiete, die beschäftigungspolitische Wirkung des Westgrenzfonds noch zu steigern, indem er an die Vergabe der Reichszuschüsse verschiedene Auflagen knüpfte. Danach mußten zur Durchführung aller Bauvorhaben, die vom Reich gefördert wurden, ortsansässige Unternehmer herangezogen werden, sofern sie den Bedingungen der Reichsverdingungsordnung entsprechen konnten. Ferner wurden alle Unternehmer, die solche Aufträge erhielten, verpflichtet, „,weitestgehend“ Material zu verwenden, das in den westlichen Grenzgebieten gewonnen oder hergestellt wurde. ${ }^{65}$

Unbeabsichtigt von den Instanzen der Wirtschaftspolitik erhöhte die schleppende Auszahlung der Fondsmittel deren Wirksamkeit bei den Anstrengungen, der hohen strukturellen Arbeitslosigkeit in den Distrikten der Westgrenze entgegenzuwirken. Bereits am 13. Juni 1928 mahnte die Zentrumsfraktion die Reichsregierung, „die sofortige Auszahlung der im Grenzfonds

${ }_{61}$ BHStA MWi 8233, 12.9.28; MWi 8231, 24.11.28, 25.3.29; MK 15 539, 6.7. 28.

62 Ebd. MWi 8231, 31.5.28; MWi 8233, 21.3., 12.9.28.

${ }^{63}$ Ebd., 29.9.28, vgl. ferner die Bestimmungen in den Antragsformularen BA R 43 I/ 1799, Bl. 109.

${ }^{64} \mathrm{Vgl}$. hierzu die Eingaben und die Prüfung von Projekten in BHStA MWi 8233 u. MA 107777.

65 BHStA MA 107776, 15.3.28; GStA Rep. 90, Nr. 1126, 3.4. 28. 
für 1928 bereitgestellten Mittel zu veranlassen, damit die vorgesehenen Bauten und sonstigen Arbeiten unverzüglich in Angriff genommen und ausgeführt werden können. "66 Staatssekretär Schmid erwiderte, die Hilfsmaßnahmen für die Westgrenze seien ,in voller Durchführung begriffen“, mußte aber eingestehen, daß ,hier und da gewisse örtliche Hemmungen“" aufgetreten seien ${ }^{67} \mathrm{Im}$ folgenden Frühjahr traten beim Vollzug des Reichshaushalts zum ersten Mal „Kassennöte“ auf. So mußte Reichsfinanzminister Hilferding im Juni 1929 dem Ministerium für die besetzten Gebiete melden, es sei ihm bei der augenblicklichen Kassenlage des Reiches leider nicht möglich, den erbetenen Betrag von $438000 \mathrm{RM}$ sofort zur Verfügung zu stellen. Er hoffe aber, die angeforderten Mittel Anfang Juli flüssig zu machen. ${ }^{68}$ Diese Verzögerung in der Abwicklung des Westgrenzfonds führte dazu, daß noch 1930 Fondsgelder ausgezahlt wurden ${ }^{69}$, deren beschäftigungspolitische Wirksamkeit nun, da sich die deutsche Wirtschaft bereits in der Krise befand, wesentlich höher war als 1928 oder auch noch 1929. Zumindest einige Bauvorhaben konnten auf diese Weise weitergeführt werden, die ohne staatliche Hilfe wohl der nachlassenden Investitionsbereitschaft während der Weltwirtschaftskrise zum Opfer gefallen wären.

66 RT 430, Nr. 7.

67 RT 423, S. 29.

68 BHStA MK 15539, 18.6.29. Zu Hilferdings Politik der Überbrückungskredite vgl. L. Mühlich, Die Reichsfinanzpolitik in der Weltwirtschaftskrise 1929 - 32 unter besonderer Berücksichtigung der Finanzpolitik der Reichsregierung Brüning, Diss. Tübingen 1950, S. $29 \mathrm{f}$.

69 BLT III, Beil. Bd. 4, S. 367; Die Pfalz unter französischer Besatzung von 1918 bis 1930 , 1930, S. 347; BA R 43 I/1842, 17.5.29. 